\title{
Suppression of Decoherence in Fast-Atom Diffraction at Surfaces
}

\author{
F. Aigner, ${ }^{1}$ N. Simonović,,${ }^{1,2}$ B. Solleder,${ }^{1}$ L. Wirtz,${ }^{3}$ and J. Burgdörfer ${ }^{1}$ \\ ${ }^{1}$ Institute for Theoretical Physics, Vienna University of Technology, A-1040 Vienna, Austria, EU \\ ${ }^{2}$ Institute of Physics, P.O. Box 57, 11001 Belgrade, Serbia

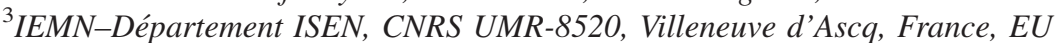

(Received 30 April 2008; published 17 December 2008)

\begin{abstract}
Scattering of fast neutral atoms with keV kinetic energies at alkali-halide surfaces under grazing angles displays intriguing diffraction patterns. The surprisingly strong persistence of quantum coherence despite the impulsive interaction with an environment at solid state density and elevated temperatures raises fundamental questions such as to the suppression of decoherence and of the quantum-to-classical crossover. We present an ab initio simulation of the quantum diffraction of fast helium beams at a $\mathrm{LiF}$ (100) surface in the $\langle 110\rangle$ direction and compare with recent experimental diffraction data. From the quantitative reconstruction of diffraction images the vertical LiF-surface reconstruction, or buckling, can be determined.
\end{abstract}

Diffraction of massive particles scattered at surfaces was one of the key experiments establishing the quantum wave nature. Coherent atom and molecule optics was initiated when Estermann and Stern [1] observed interference patterns of slow ("thermal") helium atoms and $\mathrm{H}_{2}$ molecules scattered off alkali-halide surfaces. Thermal atom scattering (TAS) $[2,3]$ as well as low-energy electron diffraction (LEED) [4] are nowadays routinely employed to accurately probe properties of surfaces. These techniques exploit the fact that the de Broglie wavelength $\lambda_{\mathrm{dB}}$ of the beam particles is comparable to the lattice constant $a$. More recently, probing the wave nature of massive objects has taken center stage in exploring the quantum-toclassical crossover $[5,6]$. One ingredient of the quantumto-classical transition is the limit of small de Broglie wave length $\lambda_{\mathrm{dB}} \rightarrow 0$, the other the ubiquitous presence of unobserved environmental degrees of freedom within the framework of decoherence theory [1], or alternatively, elements beyond standard unitary quantum dynamics, including the frequently involved "collapse" of the wave functions $[7,8]$. In matter-wave interferometry of large and complex biomolecules, de Broglie wavelengths as small as few picometer $\left(10^{-12} \mathrm{~m}\right)$ have been reached [6].

Even shorter wavelengths have been accessed by recent fast-atom scattering studies at surfaces [9-11] with $\lambda_{\mathrm{dB}}$ as small as $\approx 200$ femtometers. Persistence of quantum diffraction and the apparent suppression of decoherence is all the more remarkable, as thermal fluctuation amplitudes of the surface atoms are much larger than $\lambda_{\mathrm{dB}}$ and, moreover, collisions with $\mathrm{keV}$ projectile energies would strongly suggest the dominance of dissipative and decohering processes. In this Letter we present an $a b$ initio simulation of fast helium atom diffraction $\left({ }^{4} \mathrm{He}\right)$ at a $\operatorname{LiF}(100)$ surface. We analyze the suppression of decoherence in grazingincidence scattering within an open quantum system (OQS) approach and quantitatively reconstruct experimen- tal diffraction images $[9,10]$ in considerable detail. Fastatom diffraction has the potential to become a powerful tool to interrogate structural and dynamical properties of surfaces.

Fast-atom scattering at surfaces under small grazing angles $\left(\theta_{\text {in }} \approx 1^{\circ}\right)$ is characterized by widely disparate kinetic energies parallel [Fig. 1(a)] $\left(E_{\|}=E_{0} \cos 2 \theta_{\text {in }} \approx\right.$ $\left.E_{0}\right)$ and normal $\left(E_{\perp}=E_{0} \sin 2 \theta_{\text {in }} \approx E_{0} \theta_{\text {in }}^{2}\right)$ to the surface. This leads to vastly different projected de Broglie wavelengths

$$
\lambda_{\mathrm{dB} \perp, \|}=2 \pi / k_{\perp, \|}=2 \pi \hbar / \sqrt{2 M E_{\perp, \|}}
$$

where $k_{\|}=k_{x}$ and $k_{\perp}=k_{z}$ are parallel and normal components of the wave vector of the incident beam, respectively. While $\lambda_{\mathrm{dB} \perp}$ is comparable to the lattice spacing $(a=2.01 \AA$ for $\mathrm{LiF}) \lambda_{\mathrm{dB} \|}$ is orders of magnitude smaller. The fast motion along the surface results in a weak coupling between the longitudinal and the transverse degrees of freedom [12]. By averaging the atom-surface potential along the direction of the incident atom ( $x$ direction), the scattered particle experiences an effective twodimensional atomic string potential $V_{\text {str }}(y, z)$ [Fig. 1(c)]. Projecting the outgoing wave onto the plane transverse to the beam direction $\left|\left\langle k_{y} k_{z} \mid \Psi_{\text {out }}\right\rangle\right|^{2}$, two-dimensional diffraction patterns [see Fig. 1(a)] emerge. The remarkable persistence of quantum coherence in the presence of an energetic collision with $\mathrm{keV}$ energies and lattice vibrations at elevated temperatures in the experiment $(T \approx 620 \mathrm{~K}$ [9]) is the key to quantitatively analyzing diffraction patterns and extracting structural and dynamical information.

Starting point of the open quantum system approach $[13,14]$ is the reduction of the Liouville-von Neumann equation for the density operator $\rho$ of the entangled system of atomic wave packet and surface to a Lindblad equation 
a)

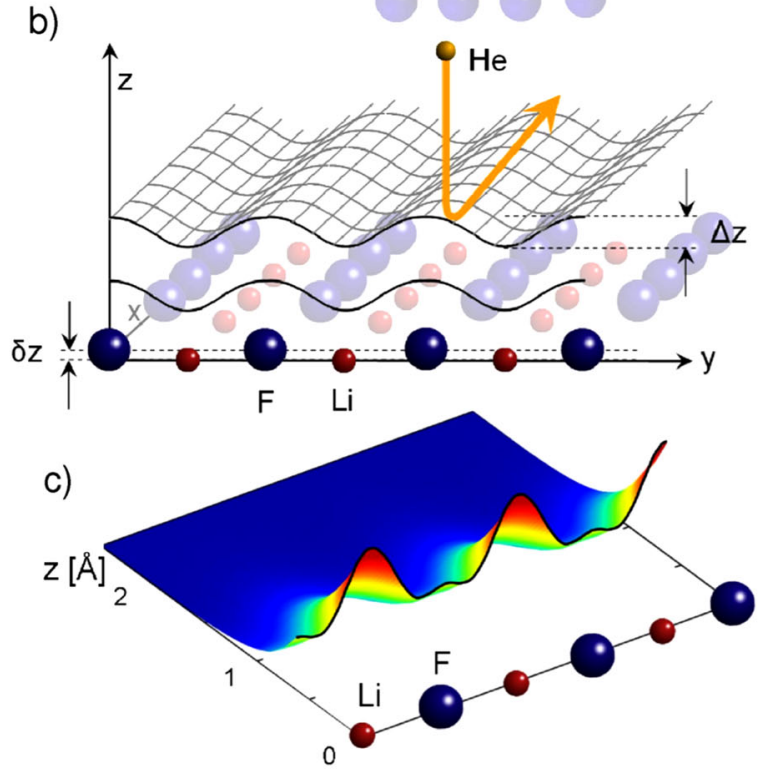

FIG. 1 (color online). Helium atoms scattered at the $\mathrm{LiF}$ surface at a grazing angle of incidence (a). In 110 direction, the $\mathrm{He}$ atoms interact with longitudinal chains of either $\mathrm{Li}$ or $\mathrm{F}$ atoms (b). Averaged effective two-dimensional string potential $V_{\text {str }}(\mathrm{c})$.

$$
d \sigma(t) / d t=-i\left[H_{S}, \sigma(t)\right]+\Re \sigma(t),
$$

for the reduced density operator $\sigma(t)=\operatorname{Tr}_{R}[\rho(t)]$, describing the translational motion of the atomic wave packet where all "reservoir" degrees of freedom $(R)$ representing the LiF surface have been traced out. This trace includes both the average over thermal excitation of the surface as well as collisional excitations of the surface by the beam by the so-called relaxation operator $\Re$. This term allows us to approximately include many-body effects beyond the single particle scattering description. The initial density matrix of the atomic beam prior to entanglement with the solid,

$$
\sigma(t=0)=\int d^{3} p_{i}\left|\Psi_{\vec{p}_{i}}\right\rangle\left\langle\Psi_{\vec{p}_{i}}\right|
$$

corresponds to an integral over wave packets representing the incoherent energy- and angular spread of the initial beam.

We solve the Lindblad equation by a quantum trajectory Monte Carlo (QTMC) method [13] which is an extension of similar techniques in quantum optics [14]. In the present case of free-particle wave packets; the QTMC simulation reduces to an ensemble average over solutions of a stochastic linear Schrödinger equation. The evolution of the wave packet is given by a sequence of collisions and propagation in the effective potential. Each stochastic realization of the wave packet at the time $t=t_{N}$ is given by

$$
\left|\Psi_{i}(t)\right\rangle=\prod_{j=1}^{N} U_{\mathrm{coll}}\left(\vec{q}_{j}, t_{j}\right) U_{\mathrm{cont}}\left(t_{j}, t_{j-1}\right)\left|\Psi_{i}\right\rangle
$$

where $\left|\Psi_{i}\right\rangle$ is the initial wave packet at $t_{0}=0$. The evolution operator for an impulsive stochastic collisional momentum exchange $\vec{q}_{j}$ with the surface is given by $U_{\text {coll }}\left(\vec{q}_{j}, t_{j}\right)=\exp \left(i \vec{q}_{j} \vec{r}\right)$, while the continuous evolution in between two "kicks" during the interval $\left[t_{j}, t_{j-1}\right]$ is given by $U_{\text {cont }}\left(t_{j}, t_{j-1}\right)=\exp \left(-i H_{S}\left(t_{j}-t_{j-1}\right)\right)$, with the system Hamiltonian

$$
H_{S}=\frac{P_{\perp}^{2}}{2 m}+V_{\mathrm{str}}(y, z)+\frac{P_{\|}^{2}}{2 m} .
$$

Momentum transfers $\left\{\vec{q}_{j}\right\}$ as well as collision times $\left\{t_{j}\right\}$ are stochastic sequences determined by collisional interactions with the environment. The resulting diffraction pattern in the transverse plane is calculated as an ensemble average over stochastic realizations.

Electronic excitations are strongly suppressed in wideband-gap insulators and energetically well separated from quasielastic collisions between neutral atoms. Electronically inelastic processes lead to "strong" decoherence or dissipation [13]. Weak decoherence originates from momentum transfers in binary $\mathrm{He}-\mathrm{F}$ and $\mathrm{He}-\mathrm{Li}$ collisions, which result in phonon excitations [15]. Their longitudinal, $P\left(q_{\|}\right)$, and transverse, $P\left(q_{\perp}\right)$, distribution functions (Fig. 2), (proportional to differential cross sections) are averaged over thermal displacement of the lattice atoms. $q_{\perp}$ displaces the momentum of each wave packet, while the effect of $q_{\|}$is more subtle: it causes velocity and kinetic energy changes of the parallel motion and depends on $\left(\lambda_{\mathrm{dB} \|}\right)^{-1}$. The energy exchange with the surface (of the order of $\approx \mathrm{eV}$ ) results in random phases in the continuous time evolution.

We determine $V_{\text {str }}(y, z)$ as the string average along the $\langle 110\rangle$ direction of the full He-LiF surface potential $V_{\text {surf }}(\vec{r})$ calculated for a large LiF cluster on the multiconfiguration self-consistent field level, using the quantum chemistry code COLumbus [16]. The string potential along the $\langle 110\rangle$ direction is periodic with $V_{\text {str }}(y+d, z)=V_{\text {str }}(y, z)$, where $d$ is the spacing between adjacent strings of F. A key parameter characterizing the diffractive scattering induced by $V_{\text {str }}$ is the corrugation amplitude of the string potential, $(\Delta z)_{\text {str }}$, i.e., the maximum variation of the normal $(z)$ coordinate of the equipotential surface $E_{\perp}=V_{\text {str }}(y, z)$. For a wide interval of normal energies $\left(0.5 \mathrm{eV} \leq E_{\perp} \leq\right.$ $10 \mathrm{eV}$ ), within which a converged potential for a large cluster can be generated, $(\Delta z)_{\text {str }}$ is remarkably weakly 


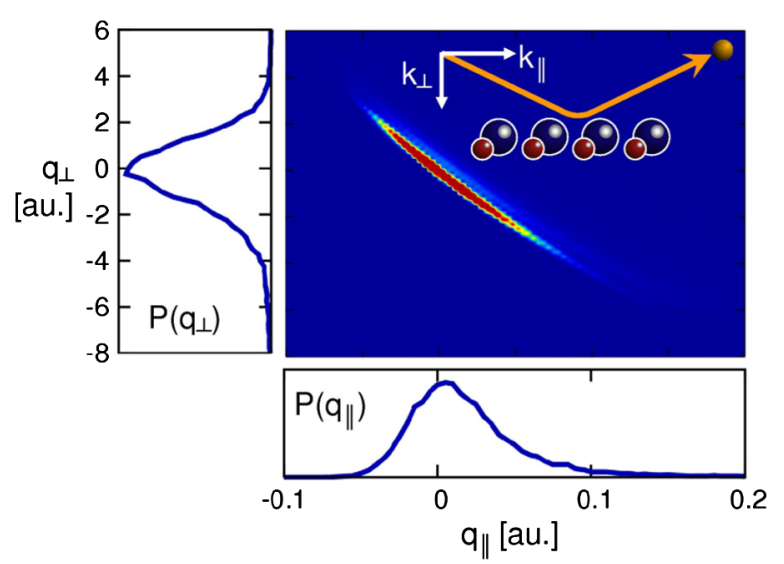

FIG. 2 (color online). Probability density function of momentum transfer of a $\mathrm{He}$ atom hitting a $\mathrm{LiF}$ surface with a temperature of $620 \mathrm{~K} . P\left(q_{\perp}\right)$ and $P\left(q_{\|}\right)$are projections onto the transverse (left) and longitudinal (bottom) direction.

dependent on $E_{\perp}$ and essentially constant $(\Delta z)_{\text {str }} \approx 0.3 \AA$.

Diffraction images can be successfully reconstructed only when decay of coherence across the beam profile due to encoding of localization information ("which path" information) is accounted for. Neglecting first the initial incoherent beam divergence, we consider an incident Gaussian wave packet (Fig. 3) of the form

$$
\Psi_{\text {in }}(\vec{r})=e^{i k_{\|} x} \Psi_{\perp}(y, z),
$$

with

$$
\Psi_{\perp}(x, y) \propto e^{-i k_{\perp} z} e^{-y^{2} / 2 \sigma_{y}^{2}} e^{-\left(z-z_{0}\right)^{2} / 2 \sigma_{z}^{2}} .
$$

$\sigma_{y, z}$ are the transverse coherence length of the incident beam determined by the width of the zeroth-order diffraction peak $\left(\sigma_{y}^{\text {in }}=\sigma_{z}^{\text {in }} \approx 2 L \frac{\lambda_{d B \|}}{D}\right)$. The latter is controlled by the collimation slit of width $D$ positioned at a distance $L$ in front of the surface. The transverse coherence length is large compared to the lattice spacing. In fact, Eq. (6) is close to a delocalized plane wave. We expand the initial wave packet in terms of a coherent superposition of narrow Gaussian wave packets [Fig. 3(a)] each of which is propagated according to Eq. (4). The random evolution phases acquired due to collisions occurring at different instances in time (i.e., different $x$ coordinates along the string) causes a reduction of the transverse coherence length [Fig. 3(b)] along the surface normal, $\sigma_{z}^{\text {out }}$, of the projected wave packet, $\left\langle\left|\Psi_{\perp}\left(y, z_{0}\right)\right|^{2}\right\rangle$ monitored after its center reached distance from the surface $z=z_{0}$ again. $\sigma_{z}^{\text {out }}$ is found to be only moderately dependent on the target temperature and the surface Debye temperature. In spite of the reduced width $\sigma_{z}^{\text {out }}$, the surface-projected length, $\sigma_{z}^{\text {out }} / \sin \theta>a$, still exceeds the lattice spacing and allows for quantum interference. Reconstruction of the experimental diffraction pattern requires, in addition, incoherent averaging over the angular divergence taken to be rectan- a)
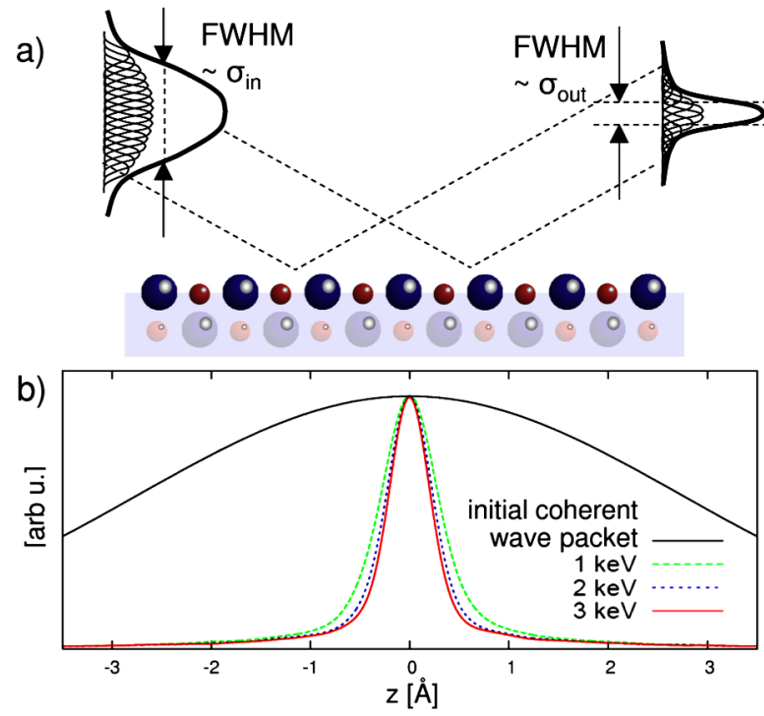

FIG. 3 (color online). A delocalized wave packet hits the surface that is considerabely wider than the size of the unit cell. Different components of the wave packet [(indicated in (a) by narrower wave packets] are subject to different random momentum changes, which lead to a partial loss of coherence. The coherent width of the wave packet is therefore drastically reduced (b).

gular, and energy spread taken to be approximately Lorentzian.

We compare the resulting diffraction patterns to experimental results [9] for scattering of He atoms $\left(E_{0}=1 \mathrm{keV}\right)$ on a $\operatorname{LiF}(001)$ surface aligned along $\langle 110\rangle$ direction under a grazing angle of incidence $\theta_{\text {in }}=1.1^{\circ}\left(E_{\perp} \approx 0.38 \mathrm{eV}\right)$. We find a near-perfect reconstruction of the experimental diffraction pattern without any adjustable parameter (Fig. 4). The positions of the maxima are obtained from the Bragg interference condition for a periodic potential along the $y$ direction, $\sin \alpha_{n}=k_{y} / k_{\perp}=n \lambda_{\mathrm{dB} \perp} / d, n=0, \pm 1, \pm 2, \ldots$ These Bragg peaks are broadened ("quasi-Bragg" peaks) due to random scattering, decoherence, and the beam divergence. The intensity modulation of the quasi-Bragg peaks is controlled by the shape of the string potential and the beam energy. The enhancement near the outermost peaks is due to the classical rainbow scattering. Additional modulations in between correspond to quantal "supernumerary" rainbows.

To demonstrate the high sensitivity of the diffraction pattern on specific features of the atomic string potential, we compare the experimental data with two different numerical simulations (Fig. 4). Strikingly, there is nearperfect agreement of the experimental modulation pattern [Fig. 4(a)] with the simulation shown in Fig. 4(b), but sizable quantitative disagreement with another, displayed in Fig. 4(c). The simulation in Fig. 4(c) uses $V_{\text {str }}$ for an ideal (unreconstructed) $\mathrm{LiF}$ surface where the $\mathrm{Li}$ and F rows are in the same plane $(z=0)$, while Fig. 4(b) is calculated for a surface with "buckling," i.e., vertical surface reconstruction where the $\mathrm{F}$ atoms are displaced 
a)

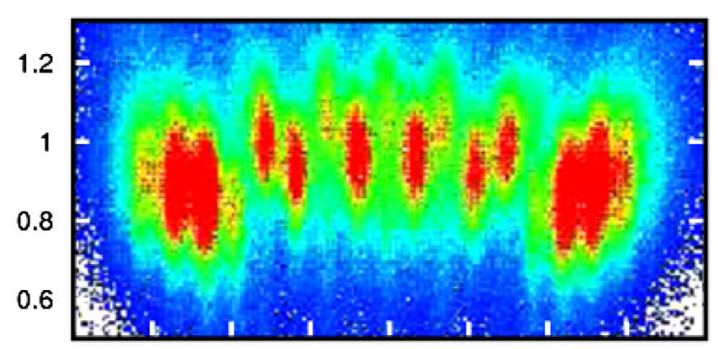

b)
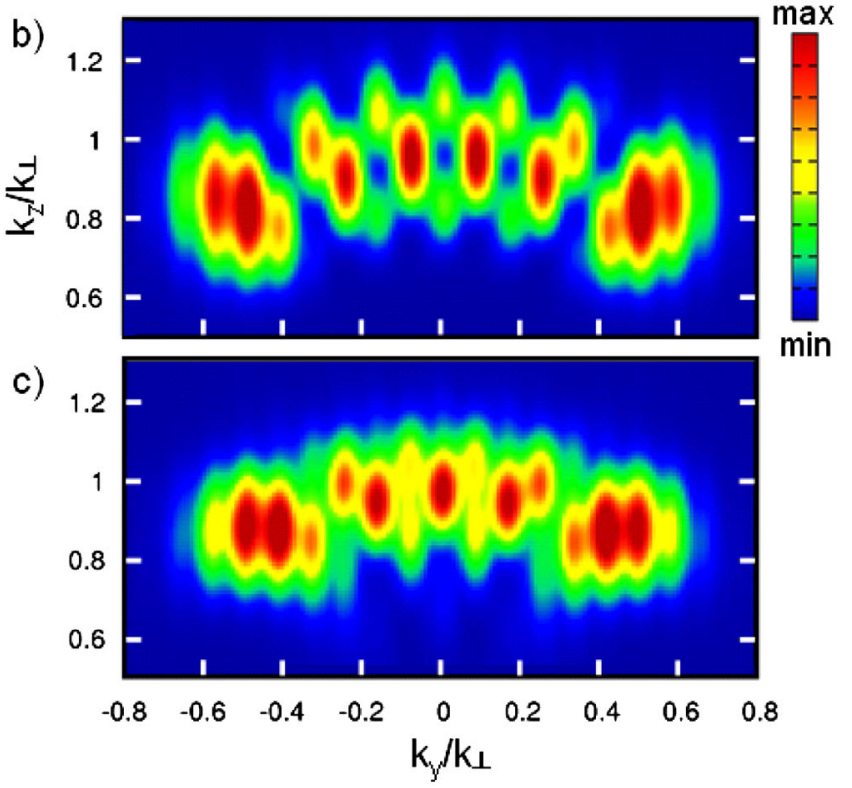

FIG. 4 (color online). Experimental (from Ref. [9]) (a) and theoretical (b),(c) diffraction patterns for scattering of $1 \mathrm{keV} \mathrm{He}$ from LiF under $\theta=1.1^{\circ}$. In (b), with surface buckling $\delta z=$ $0.053 \AA$, (c) without surface buckling $\delta z=0$. Note the different number of interference maxima.

relative to the Li atoms outward by small amount of $\delta z=$ $0.053 \AA$. This buckling amplitude was determined by a density functional theory (DFT) calculation and agrees quantitatively with earlier estimates using electrostatic shell models [18]. Pioneering experimental investigations of the $\operatorname{LiF}(100)$ surface structure employing LEED [19] also suggested the presence of a small surface reconstruction. However, experimental uncertainties were of the same size as the displacement amplitude itself and, thus, buckling could not unambiguously be established. Note that the buckling amplitude $\delta z$ is only $\approx 16 \%$ of the size of the potential surface corrugation $(\Delta z)_{\text {str }}$ of the unreconstructed surface and even much smaller than the thermal vibration amplitude. Yet, the inclusion of buckling drastically and even quantitatively changes the diffraction pattern (enhancement of odd supernumeraries in $b$, enhancement of even supernumeraries in $c$ ) and may allow to determine $\delta z$ with improved accuracy.

Summarizing, fast $\mathrm{keV}$ atomic collisions with insulating alkali-halide surfaces give rise to remarkably sharp and well-resolved diffraction images. Despite de Broglie wave- lengths as small as $100 \mathrm{fm}$, quantum interference persists. Because of the weak coupling of transverse and longitudinal degrees of freedom decoherence is effectively suppressed in grazing incidence collisions. $A b$ initio quantum trajectory Monte Carlo simulations accurately reproduce the diffraction pattern and allow to extract structural surface information such as buckling at an unprecedented level of detail.

We thank H. Winter and A. Schüller (Berlin) for helpful discussions. This work was supported by the FWF-Austria, SFB016 and P17359, and by the EU HPRI-CT-2005026015 (ITS-LEIF).

[1] I. Estermann and O. Stern, Z. Phys. 61, 95 (1930).

[2] Y. Ekinci and J.P. Toennies, Surf. Sci. 563, 127 (2004), and references therein.

[3] D. J. Riley et al., J. Chem. Phys. 126, 104702 (2007) and references therein.

[4] M. A. van Hove, W. H. Weinberg, and C.-M. Chan, LowEnergy Electron Diffraction (Springer-Verlag, Berlin, 1986).

[5] M. Arndt et al., Nature (London) 401, 680 (1999); K. Hornberger et al., Phys. Rev. Lett. 90, 160401 (2003).

[6] L. Hackermüller et al., Phys. Rev. Lett. 91, 090408 (2003).

[7] W. H. Zurek, Phys. Today 44, No. 10, 36 (1991).

[8] G. C. Ghirardi, A. Rimini, and T. Weber, Phys. Rev. D 34, 470 (1986).

[9] A. Schüller, S. Wethekam, and H. Winter, Phys. Rev. Lett. 98, 016103 (2007).

[10] A. Schüller and H. Winter, Phys. Rev. Lett. 100, 097602 (2008).

[11] P. Rousseau et al., Phys. Rev. Lett. 98, 016104 (2007).

[12] J. Burgdörfer, in Review of Fundamental Processes and Applications of Atoms and Ions, edited by C.D. Lin (World Scientific, Singapore, 1993).

[13] T. Minami, C. O. Reinhold, and J. Burgdörfer, Phys. Rev. A 67, 022902 (2003); M. Seliger et al., Phys. Rev. A 75, 032714 (2007), and references therein.

[14] J. Dalibard, Y. Castin, and K. Mølmer, Phys. Rev. Lett. 68, 580 (1992); R. Dum, P. Zoller, and H. Ritsch, Phys. Rev. A 45, 4879 (1992).

[15] A. Mertens and H. Winter, Phys. Rev. Lett. 85, 2825 (2000); R. Pfandzelter, A. Mertens, and H. Winter, Phys. Lett. A 290, 145 (2001); J. G. Roberts, M. A. van Hove, and G. A. Somorjai, Surf. Sci. 518, 49 (2002).

[16] H. Lischka et al., computer code COLUMBUS, Release 5.9 (2006).

[17] As some of the experimental diffraction data are taken at even smaller perpendicular energies for $\left(E_{\perp}<0.5 \mathrm{eV}\right)$, where the ab initio potential calculation becomes unreliable, we have extrapolated $V_{\text {str }}$ to low-energy surfaces subject to the constraint that $(\Delta z)_{\text {str }}$ remains constant. Imposition of such a constraint has proven remarkably successful when compared with experimental data.

[18] F. W. de Wette, W. Kress, and U. Schröder, Phys. Rev. B 32, 4143 (1985).

[19] J. Vogt and H. Weiss, Surf. Sci. 501, 203 (2002). 